\title{
An alternative design method for the double-layer combined die using autofrettage theory
}

\author{
Chengliang Hu, Fengyu Yang, Zhen Zhao, and Fan Zeng \\ Institute of Forming Technology \& Equipment, Shanghai Jiaotong University, Shanghai 200030, China \\ Correspondence to: Zhen Zhao (zzhao@ sjtu.edu.cn)
}

Received: 4 October 2016 - Revised: 6 June 2017 - Accepted: 26 July 2017 - Published: 28 August 2017

\begin{abstract}
The double-layer combined die is used for its longer life in forging. Autofrettage is a well-known elastic-plastic technology that increases the durability of thick-walled cylinders. This study explores an alternative design method of the double-layer combined die using autofrettage theory. An analytical solution for the autofrettage process of the double-layer combined die is obtained based on Lamé's equation. The relationship between the autofrettage pressure and the yield radius of the die insert is obtained, and expressions of residual stresses and displacements, which are directly related to geometric parameters, material properties and internal pressure, are derived. The finite-element simulation of a specific case is performed, and good agreement between theoretical calculations and simulation results is found. Furthermore, the effects of important parameters, including the ratio of the plastic area and yield strength of the die insert and the outer diameters of the die insert and stress ring, on the autofrettage effect are investigated. Compared with the conventional combined die, the autofrettaged die can bear larger working pressure, as expected. The use of the autofrettaged die can reduce the amount of expensive material required for the die insert and the working space of the die set, which would benefit the practical forging process.
\end{abstract}

\section{Introduction}

In the process of cold forging, a die usually needs to be of high strength to resist a large forging load. The accumulation of plastic deformation near points of stress concentration resulting from the cyclic loading conditions leads to fatigue damage and eventually to the generation of a crack on the surface of the die (Pedersen, 2000). A die insert with one or more massive stress rings, which is called a combined die or prestressed die, is thus designed to reduce the stress level during forging.

High radial and circumferential stresses strongly affect the elastic deformation and failure of forging dies, and the time and economic losses due to unpredicted die failure during service in forging are costly. Against this background, many studies (Joun et al., 2002; Yeo et al., 2001; Kwan and Wang, 2011; Yang et al., 2012) have attempted to optimize the dimensions of the die structure employing different optimization methods.

Usually, high-strength tool steel is used for the die insert, while normal steel is used for the stress rings. It was found that a backward extrusion die prestressed with sintered carbide could be strengthened (Hur et al., 2002) and reduce elastic deformation (Hur et al., 2003). The strip-wound container with a winding core of tungsten carbide made it possible to create new innovative die designs (Groenbaek and Birker, 2000). The cited works introduced the stress ring with highstiffness material or structure that could strengthen the combined die effectively, but the cost of a single set of the combined die increased.

Autofrettage is a well-known elastic-plastic technology that increases the durability of thick-walled cylinders, and is commonly used in high-pressure vessels. A cylinder tube is subjected to uniform internal pressure so that its wall becomes partially plastic, resulting in internal compressive residual stresses that increase the pressure capacity for the next loading (Majzoobi et al., 2003). The optimum autofrettage pressure is not constant but depends on the working pressure (Hojjati and Hassani, 2007). To improve the performance of a type-III hydrogen pressure vessel, the most appropriate autofrettage pressure has been determined by 
finite-element (FE) simulation (Son et al., 2012). The pressure reached in available commercial autofrettage processes is between 370 and $1200 \mathrm{MPa}$ (http://www.felss.com).

A method of calculating the stress intensity factor was used to estimate the fatigue life of an autofrettage tube, and results showed that the fatigue life increased with the autofrettage level (Jahed et al., 2006). The ultimate load bearing capacity of the autofrettage cylinder was estimated theoretically (Zhu, 2008a, b). The autofrettage process led to tangible increases in the strength-to-weight ratio and fatigue life of the cannon tube (Anantharam and Kumar, 2014). A novel concept of an autofrettage compounded tube was proposed to give a high safe pressure and good fatigue life (Bhatnagar, 2013). Therefore, using autofrettage technology, the load bearing capacity, strength-to-weight ratio and fatigue life of cylinder parts can be improved.

Considering the advantages of autofrettage technology, it is desirable to explore the possibility of the application of the technology to the cold forging die. The effect law of strain hardening and the Bauschinger effect on the autofrettage process have been discussed by comparing the results of a bilinear kinematic hardening model and ideal elastoplastic model, and the optimum autofrettage pressure was determined to meet the high dimensional accuracy and strength requirements of a single-layer extrusion die (Qian et al., 2011). However, the single-layer die is seldom used in actual forging.

The present study obtained an analytical solution for the autofrettage process of the double-layer combined die and established formulae of the residual stresses and displacements. The simulation results for a specific case of the double-layer combined die after autofrettage were used to verify the reliability and accuracy of the theoretical derivation. On this basis, other cases were considered in studying the effects of the ratio of the plastic area and yield strength of the die insert and the outer diameters of the die insert and stress ring on the autofrettaged die. The results clarify the autofrettage effect and contribute to the design of the doublelayer combined die for forging.

Different from previous design strategies - such as using stronger material for the die insert directly, optimization of the die dimensions including the interference fit, and using a stress ring with a high-stiffness material or structure - a novel design method of the combined die is proposed, where compressive stresses on the die insert are introduced by the autofrettage process to improve the strength of the combined die.

\section{Theory and formulation}

The combined die, which consists of insert die and stress ring, is first strengthened by the classical shrink fit, and the autofrettage process is then applied as a pre-process before the combined die is used in forging. During the autofrettage process, an enormous uniform internal pressure is loaded on the double-layer combined die, so that there is uniform plastic deformation of the die. After unloading, there are residual stresses and a plastic area with certain thickness emerges. A theoretical discussion of the autofrettage process is now presented.

Several assumptions are made. (1) The double-layer combined die can be regarded as an axially constrained cylinder. (2) Because of the small axial deformation, the autofrettage process can be simplified as a plane-strain problem. (3) The material of the die insert is a perfectly elastic-plastic material and obeys the von Mises criterion. (4) The material is supposed to be incompressible. (5) Plastic deformation only occurs in the die insert, while the stress ring remains in the elastic state.

\subsection{Stress in the loading process}

In the loading process, the die insert can be subdivided as inner-plastic and outer-elastic layers considering the elasticplastic deformation behavior of the combined die. The uniform autofrettage pressure is denoted $p_{0}$. The radius at the boundary between plastic and elastic areas is referred to as the yield radius $r_{p}$, the normal stress between the two areas is denoted $q$, and the normal stress between die insert and stress ring is denoted $p_{k}^{p}$, as shown in Fig. 1 .

According to the equilibrium equation and the yield criterion

$\frac{\mathrm{d} \sigma_{r}}{\mathrm{~d} r}-\frac{\sigma_{s}}{r}=0$.

Under the assumptions mentioned above, the radial stress in the inner-plastic layer can be obtained according to the equilibrium, Eq. (1):

$\sigma_{r}=\frac{2}{\sqrt{3}} \sigma_{s} \ln r+C\left(r_{1} \leq r \leq r_{p}\right)$.

The constant $C$ can be calculated using the boundary condition $\sigma_{r}=-p_{0}$ when $r=r_{1}$ :

$C=-p_{0}-\frac{2}{\sqrt{3}} \sigma_{s} \ln r_{1}$.

Then, $\sigma_{r}$ in the plastic area of the die insert can then be solved as

$\sigma_{r}=\frac{2}{\sqrt{3}} \sigma_{s} \ln \frac{r}{r_{1}}-p_{0}\left(r_{1} \leq r \leq r_{p}\right)$.

and $\sigma_{\theta}$ in the plastic area of the die insert can be solved as

$\sigma_{\theta}=\sigma_{r}+\sigma_{s}=\frac{2}{\sqrt{3}} \sigma_{s}\left(1+\ln \frac{r}{r_{1}}\right)-p_{0}\left(r_{1} \leq r \leq r_{p}\right)$.

According to the Eqs. (4) and (5), the stress components of plastic area are stationary (Xu and Liu, 1995), and they are 


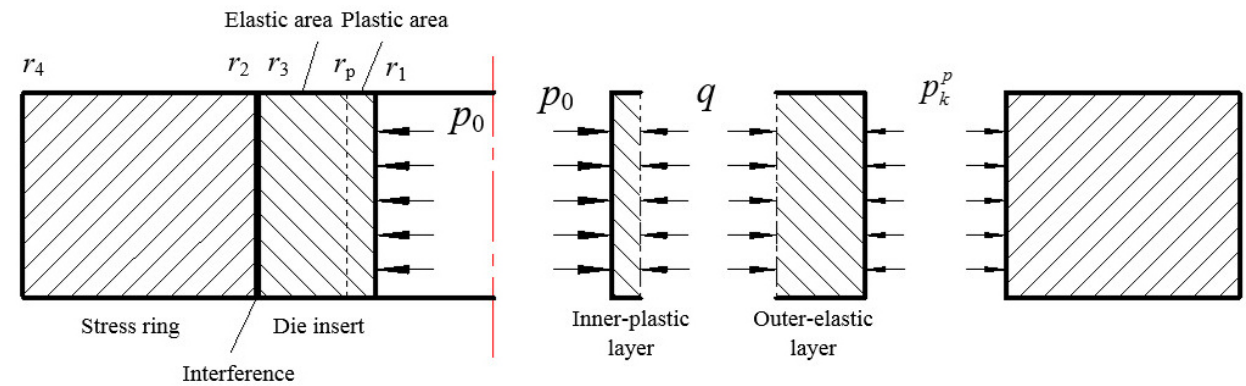

Figure 1. Double-layer combined die.

only related to internal pressure $p_{0}$, and has nothing to do with the stress of elastic area.

As shown in Fig. 1, the outer-elastic layer can be regarded as a thick cylinder under internal pressure $q$ and external pressure. Using Lamé's formula, the external pressure is obtained according to the geometry boundary condition $r_{2}+u_{2}=r_{3}+u_{3}$, where $u_{2}$ and $u_{3}$ are the displacements due to the interference fit between the die insert and stress ring. The external pressure is given by

$p_{k}^{p}=\frac{r_{3}-r_{2}-q \Delta_{1}^{p}}{\Delta_{2}^{p}-\Delta_{3}}$,

where $\Delta_{1}^{p}=2 r_{2} \frac{\left(1-\mu_{1}^{2}\right) r_{p}^{2}}{E_{1}\left(r_{2}^{2}-r_{p}^{2}\right)}, \Delta_{2}^{p}=\frac{\left(1+\mu_{1}\right) r_{2}^{2}}{E_{1}\left(r_{2}^{2}-r_{p}^{2}\right)}\left[\left(2 \mu_{1}-1\right) r_{2}-\frac{r_{p}^{2}}{r_{2}}\right]$ and $\Delta_{3}=\frac{\left(\mu_{2}+1\right) r_{3}^{2}}{E_{2}\left(r_{4}^{2}-r_{3}^{2}\right)}\left[\left(1-2 \mu_{2}\right) r_{3}+\frac{r_{4}^{2}}{r_{3}}\right], E_{1}$ and $E_{2}$ are respectively the elastic moduli of the materials of the die insert and stress ring, and $\mu_{1}$ and $\mu_{2}$ are respectively the Poisson ratios of the materials of the die insert and stress ring.

According to Lamé's equation, the stresses in the elastic area of the die insert are

$\left\{\begin{array}{l}\sigma_{r}=\frac{r_{p}^{2} r_{2}^{2}\left(p_{k}^{p}-q\right)}{r_{2}^{2}-r_{p}^{2}} \cdot \frac{1}{r^{2}}+\frac{r_{p}^{2} q-r_{2}^{2} p_{k}^{p}}{r_{2}^{2}-r_{p}^{2}} \\ \sigma_{\theta}=-\frac{r_{p}^{2} r_{2}^{2}\left(p_{k}^{p}-q\right)}{r_{2}^{2}-r_{p}^{2}} \cdot \frac{1}{r^{2}}+\frac{r_{p}^{2} q-r_{2}^{2} p_{k}^{p}}{r_{2}^{2}-r_{p}^{2}}\end{array}\left(r_{p} \leq r \leq r_{2}\right)\right.$,

and the stresses of the stress ring are

$\left\{\begin{array}{l}\sigma_{r}=-\frac{r_{3}^{2} r_{4}^{2} p_{k}^{p}}{r_{4}^{2}-r_{3}^{2}} \cdot \frac{1}{r^{2}}+\frac{r_{3}^{2} p_{k}^{p}}{r_{4}^{2}-r_{3}^{2}} \\ \sigma_{\theta}=\frac{r_{3}^{2} r_{4}^{2} p_{k}^{p}}{r_{4}^{2}-r_{3}^{2}} \cdot \frac{1}{r^{2}}+\frac{r_{3}^{2} p_{k}^{p}}{r_{4}^{2}-r_{3}^{2}}\end{array}\left(r_{3} \leq r \leq r_{4}\right)\right.$.

In the outer-elastic layer, the die material just yields at the inner boundary. Therefore, the normal stress can be derived according to Lamé's equation:

$$
\begin{aligned}
& q=\frac{\sigma_{s}\left(r_{2}^{2}-r_{p}^{2}\right)}{\sqrt{3} r_{2}^{2}}+p_{k}^{p} \\
& =\left(\frac{\sigma_{s}\left(r_{2}^{2}-r_{p}^{2}\right)}{\sqrt{3} r_{2}^{2}}+\frac{r_{3}-r_{2}}{\Delta_{2}^{p}-\Delta_{3}}\right) /\left(1+\frac{\Delta_{1}^{p}}{\Delta_{2}^{p}-\Delta_{3}}\right) .
\end{aligned}
$$

In the inner-plastic layer, the normal stress at the outer boundary can be solved by substituting $\sigma_{r}=-q$ into Eq. (3) when $r=r_{p}$ :

$q=-\frac{2}{\sqrt{3}} \sigma_{s} \ln \frac{r_{p}}{r_{1}}+p_{0}$.

The normal stress between the inner-plastic and outer-elastic layers should be the same. The relationship between the autofrettage pressure $p_{0}$ and the yield radius $r_{p}$ can then be obtained as

$$
\begin{aligned}
& p_{0}=\left(\frac{\sigma_{s} r_{p}^{2}\left(r_{2}^{2}-r_{p}^{2}\right)}{\sqrt{3} r_{p}^{2} r_{2}^{2}}+\frac{r_{3}-r_{2}}{\Delta_{2}^{p}-\Delta_{3}}\right) /\left(1+\frac{\Delta_{1}^{p}}{\Delta_{2}^{p}-\Delta_{3}}\right) \\
& +\frac{2}{\sqrt{3}} \sigma_{s} \ln \frac{r_{p}}{r_{1}} .
\end{aligned}
$$

\subsection{Displacement in the loading process}

According to Lamé's equation, the displacement of the stress ring is

$$
\begin{aligned}
u= & \frac{1+\mu_{2}}{E_{2}}\left[\frac{r_{3}^{2} r_{4}^{2} p_{k}^{p}}{r_{4}^{2}-r_{3}^{2}} \frac{1}{r}\right. \\
& \left.+\left(1-2 \mu_{2}\right) \frac{r_{3}^{2} p_{k}^{p}}{r_{4}^{2}-r_{3}^{2}} r\right]
\end{aligned}
$$

and the displacement in the outer-elastic layer of the die insert is

$$
\begin{aligned}
& u=\frac{1+\mu_{1}}{E_{1}}\left[-\frac{r_{p}^{2} r_{2}^{2}\left(p_{k}^{p}-q\right)}{r_{2}^{2}-r_{p}^{2}} \frac{1}{r}\right. \\
& \left.+\left(1-2 \mu_{1}\right) \frac{r_{p}^{2} q-r_{2}^{2} p_{k}^{p}}{r_{2}^{2}-r_{p}^{2}} r\right]
\end{aligned}
$$

By considering the incompressible condition and geometric equation in the plane-strain problem, the displacement in the inner-plastic layer is obtained as

$u=\frac{C_{1}}{r}\left(r_{1} \leq r \leq r_{p}\right)$. 
The displacement along the radius of the die insert should be continuous, and the displacements at the boundary between the inner-plastic and outer-elastic layers should thus be equal. When $r=r_{p}$, the displacement calculated using Eqs. (12) and (13) should be the same. The constant $C_{1}$ can then be determined as

$$
\begin{aligned}
\mathrm{C}_{1}= & \frac{1+\mu_{1}}{E_{1}}\left[-\frac{r_{p}^{2} r_{2}^{2}\left(p_{k}^{p}-q\right)}{r_{2}^{2}-r_{p}^{2}}\right. \\
& \left.+\left(1-2 \mu_{1}\right) \frac{r_{p}^{2} q-r_{2}^{2} p_{k}^{p}}{r_{2}^{2}-r_{p}^{2}} r_{p}^{2}\right] .
\end{aligned}
$$

The displacement in the inner-plastic layer of the die insert is thus

$$
\begin{aligned}
u= & \frac{1+\mu_{1}}{E_{1} r}\left[-\frac{r_{p}^{2} r_{2}^{2}\left(p_{k}^{p}-q\right)}{r_{2}^{2}-r_{p}^{2}}\right. \\
& \left.+\left(1-2 \mu_{1}\right) \frac{r_{p}^{2} q-r_{2}^{2} p_{k}^{p}}{r_{2}^{2}-r_{p}^{2}} r_{p}^{2}\right]
\end{aligned}
$$

\subsection{Residual stress after unloading}

Residual stress is the difference between stresses of the loading and unloading processes:

$\sigma_{i j}^{r}=\sigma_{i j}-\sigma_{i j}^{e}$

where $\sigma_{i j}^{r}$ denotes the residual stresses, $\sigma_{i j}$ denotes the stresses of the loading process and $\sigma_{i j}^{e}$ denotes the stresses of the unloading process.

During the unloading process, the stresses in the die insert are$$
\left\{\begin{array}{l}
\sigma_{r}^{e}=\frac{r_{1}^{e^{2}} r_{2}^{e^{2}}\left(p_{k}-p_{0}\right)}{r_{2}^{e^{2}}-r_{1}^{e^{2}}} \cdot \frac{1}{r^{2}}+\frac{r_{1}^{e^{2}} p_{0}-r_{2}^{e^{2}} p_{k}}{r_{2}^{e^{2}}-r_{1}^{e^{2}}} \\
\sigma_{\theta}^{e}=-\frac{r_{1}^{e^{2}} r_{2}^{e^{2}}\left(p_{k}-p_{0}\right)}{r_{2}^{e^{2}}-r_{1}^{e^{2}}} \cdot \frac{1}{r^{2}}+\frac{r_{1}^{e^{2}} p_{0}-r_{2}^{e^{2}} p_{k}}{r_{2}^{e^{2}}-r_{1}^{e^{2}}},
\end{array}\right.
$$

where $\quad p_{k}=\frac{r_{3}^{e}-r_{2}^{e}-p_{0} \Delta_{1}^{e}}{\Delta_{2}^{e}-\Delta_{3}^{e}}, \quad \Delta_{1}^{e}=2 r_{2}^{e} \frac{\left(1-\mu_{1}^{2}\right) r_{1}^{e^{2}}}{E_{1}\left(r_{2}^{e^{2}}-r_{1}^{e^{2}}\right)}$, $\Delta_{2}^{e}=\frac{\left(1+\mu_{1}\right) r_{2}^{e^{2}}}{E_{1}\left(r_{2}^{e^{2}}-r_{1}^{e^{2}}\right)}\left[\left(2 \mu_{1}-1\right) r_{2}^{e}-\frac{r_{1}^{e^{2}}}{r_{2}^{e}}\right], \Delta_{3}^{e}=\frac{\left(\mu_{2}+1\right) r_{3}^{e^{2}}}{E_{2}\left(r_{4}^{\left.e^{2}-r_{3}^{e^{2}}\right)}\right.}[(1-$

$\left.\left.2 \mu_{2}\right) r_{3}^{e}+\frac{r_{4}^{e^{2}}}{r_{3}^{e}}\right]$ and $r_{i}^{e}=r_{i}+u_{i}(i=1,2,3,4) . u_{i}$ is the displacement due to the interference fit between the die insert and stress ring.

The residual stresses in the die insert are then

$$
\sigma_{r}^{r}=\left\{\begin{array}{l}
\left(\frac{2}{\sqrt{3}} \sigma_{s} \ln \frac{r}{r_{1}^{e}}-p_{0}\right) \\
-\left[\frac{r_{1}^{e^{2}} r_{2}^{e^{2}}\left(p_{k}-p_{0}\right)}{r_{2}^{e^{2}}-r_{1}^{e^{2}}} \cdot \frac{1}{r^{2}}+\frac{r_{1}^{e^{2}} p_{0}-r_{2}^{e^{2}} p_{k}}{r_{2}^{e^{2}}-r_{1}^{e^{2}}}\right]\left(r_{1}^{e} \leq r \leq r_{p}^{e}\right) \\
{\left[\frac{r_{p}^{e^{2}} r_{2}^{e^{2}}\left(p_{k}-q\right)}{r_{2}^{e^{2}}-r_{p}^{e^{2}}} \cdot \frac{1}{r^{2}}+\frac{r_{p}^{e^{2}} q-r_{2}^{e^{2}} p_{k}}{r_{2}^{e^{2}}-r_{p}^{e^{2}}}\right]} \\
-\left[\frac{r_{1}^{e^{2}} r_{2}^{e^{2}}\left(p_{k}-p_{0}\right)}{r_{2}^{e^{2}}-r_{1}^{e^{2}}} \cdot \frac{1}{r^{2}}+\frac{r_{1}^{e^{2}} p_{0}-r_{2}^{e^{2}} p_{k}}{r_{2}^{e^{2}}-r_{1}^{e^{2}}}\right]\left(r_{p}^{e} \leq r \leq r_{2}^{e}\right) .
\end{array}\right.
$$

$$
\sigma_{\theta}^{r}=\left\{\begin{array}{l}
{\left[\frac{2}{\sqrt{3}} \sigma_{s}\left(1+\ln \frac{r}{r_{1}^{e}}\right)-p_{0}\right]} \\
-\left[-\frac{r_{1}^{e^{2}} r_{2}^{e^{2}}\left(p_{k}-p_{0}\right)}{r_{2}^{e^{2}}-r_{1}^{e^{2}}} \cdot \frac{1}{r^{2}}+\frac{r_{1}^{e^{2}} p_{0}-r_{2}^{e^{2}} p_{k}}{r_{2}^{e^{2}}-r_{1}^{e^{2}}}\right]\left(r_{1}^{e} \leq r \leq r_{p}^{e}\right) \\
{\left[-\frac{r_{p}^{e^{2}} r_{2}^{e^{2}}\left(p_{k}-q\right)}{r_{2}^{e^{2}}-r_{p}^{e^{2}}} \cdot \frac{1}{r^{2}}+\frac{r_{p}^{e^{2}} q-r_{2}^{e^{2}} p_{k}}{r_{2}^{e^{2}}-r_{p}^{e^{2}}}\right]} \\
-\left[-\frac{r_{1}^{e^{2}} r_{2}^{e^{2}}\left(p_{k}-p_{0}\right)}{r_{2}^{e^{2}}-r_{1}^{e^{2}}} \cdot \frac{1}{r^{2}}+\frac{r_{1}^{e^{2}} p_{0}-r_{2}^{e^{2}} p_{k}}{r_{2}^{e^{2}}-r_{1}^{e^{2}}}\right]\left(r_{p}^{e} \leq r \leq r_{2}^{e}\right) .
\end{array}\right.
$$

The stresses in the stress ring during unloading can be expressed as

$$
\left\{\begin{array}{l}
\sigma_{r}^{e}=-\frac{r_{3}^{e^{2}} r_{4}^{e^{2}} p_{k}}{r_{4}^{e^{2}}-r_{3}^{e^{2}}} \cdot \frac{1}{r^{2}}+\frac{r_{3}^{e^{2}} p_{k}}{r_{4}^{e^{2}}-r_{3}^{e^{2}}} \\
\sigma_{\theta}^{e}=\frac{r_{3}^{e^{2}} r_{4}^{e^{2}} p_{k}}{r_{4}^{e^{2}}-r_{3}^{e^{2}}} \cdot \frac{1}{r^{2}}+\frac{r_{3}^{e^{2}} p_{k}}{r_{4}^{e^{2}}-r_{3}^{e^{2}}} .
\end{array}\right.
$$

The corresponding residual stresses are

$$
\left\{\begin{array}{l}
\sigma_{r}=\left(-\frac{r_{3}^{e^{2}} r_{4}^{e^{2}} p_{k}^{p}}{r_{4}^{e^{2}}-r_{3}^{e^{2}}} \cdot \frac{1}{r^{2}}+\frac{r_{3}^{e^{2}} p_{k}^{p}}{r_{4}^{e^{2}}-r_{3}^{e^{2}}}\right) \\
-\left(-\frac{r_{3}^{e^{2}} r_{4}^{e^{2}} p_{k}}{r_{4}^{e^{2}}-r_{3}^{e^{2}}} \cdot \frac{1}{r^{2}}+\frac{r_{3}^{e^{2}} p_{k}}{r_{4}^{e^{2}}-r_{3}^{e^{2}}}\right) \\
\sigma_{\theta}=\left(\frac{r_{3}^{e^{2}} r_{4}^{e^{2}} p_{k}^{p}}{r_{4}^{e^{2}}-r_{3}^{e^{2}}} \cdot \frac{1}{r^{2}}+\frac{r_{3}^{e^{2}} p_{k}^{p}}{r_{4}^{e^{2}}-r_{3}^{e^{2}}}\right) \\
-\left(\frac{r_{3}^{e^{2}} r_{4}^{e^{2}} p_{k}}{r_{4}^{e^{2}}-r_{3}^{e^{2}}} \cdot \frac{1}{r^{2}}+\frac{r_{3}^{e^{2}} p_{k}}{r_{4}^{e^{2}}-r_{3}^{e^{2}}}\right) .
\end{array}\right.
$$

\subsection{Residual displacement after unloading}

The residual displacement is the difference between displacements of loading and unloading processes:

$u^{r}=u-u^{e}$,

where $u^{r}$ is the residual displacement, $u$ is the displacement of the loading process and $u^{e}$ is the displacement of the unloading process.

During the unloading process, the displacement in the die insert is

$$
\begin{aligned}
u^{e}= & \frac{1+\mu_{1}}{E_{1}}\left[-\frac{r_{1}^{e^{2}} r_{2}^{e^{2}}\left(p_{k}-p_{0}\right)}{r_{2}^{e^{2}}-r_{1}^{e^{2}}} \frac{1}{r}\right. \\
& \left.+\left(1-2 \mu_{1}\right) \frac{r_{1}^{e^{2}} p_{0}-r_{2}^{e^{2}} p_{k}}{r_{2}^{e^{2}}-r_{1}^{e^{2}}} r\right]
\end{aligned}
$$

The displacement in the stress ring during the unloading process is

$$
u^{e}=\frac{1+\mu_{2}}{E_{2}}\left[\frac{r_{3}^{e^{2}} r_{4}^{e^{2}} p_{k}}{r_{4}^{e^{2}}-r_{3}^{e^{2}}} \frac{1}{r}+\left(1-2 \mu_{2}\right) \frac{r_{3}^{e^{2}} p_{k}}{r_{4}^{e^{2}}-r_{3}^{e^{2}}} r\right] .
$$



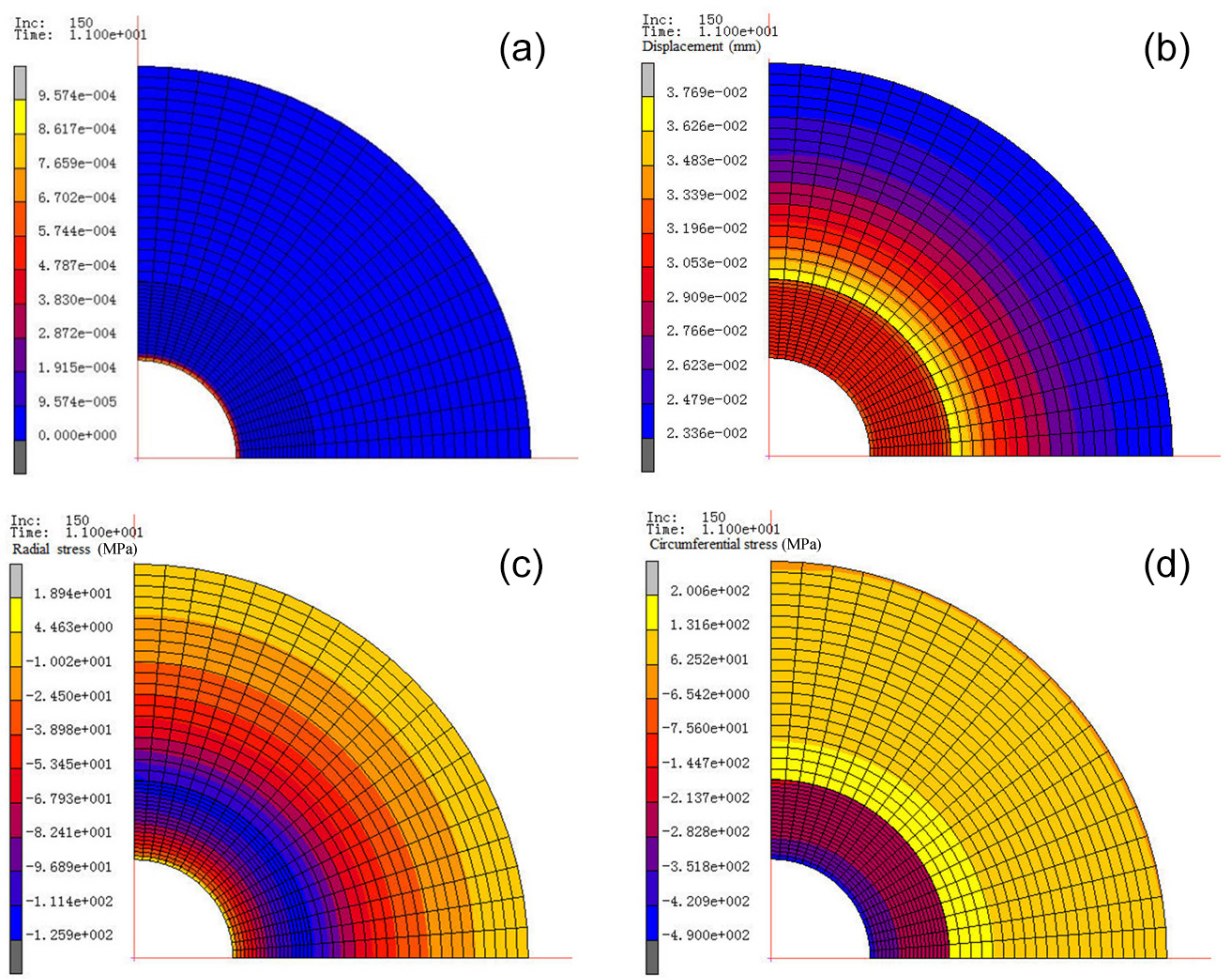

Figure 2. Distributions of (a) equivalent plastic strain, (b) residual displacement, (c) radial residual stress and (d) circumferential residual stress obtained from FE simulation results.

Table 1. Parameters of the combined die.

\begin{tabular}{lrr}
\hline Parameters & Die insert & Stress ring \\
\hline Elastic modulus (GPa) & 212 & 212 \\
Yield strength (MPa) & 1300 & 900 \\
Poisson's ratio & 0.3 & 0.3 \\
Inner diameter (mm) & 20.00 & 36.00 \\
External diameter (mm) & 36.00 & 80.00 \\
Interference fit (mm) & \multicolumn{2}{c}{0.07} \\
\hline
\end{tabular}

\section{FE simulation}

To verify the reliability and accuracy of the theoretical derivation, a specific case of a double-layer combined die strengthened by the autofrettage process is analyzed in an FE simulation. The relative parameters of the combined die are listed in Table 1. In the simulated case, the autofrettage pressure is $940.0 \mathrm{MPa}, 5.0 \%$ of the die insert undergoes plastic deformation and the yield radius is $20.80 \mathrm{~mm}$.

According to the parameters given above, a 1/4-scale geometric model was constructed using the commercial simulation software MSC MARC. The material model obeys elastic-plastic law perfectly. A total of 800 full integration quad elements were used and no remeshing was triggered during the simulation. During the autofrettage process, the combined die was first subjected to inner pressure of 940.0 MPa until there was partial plasticity, and the pressure was then released to $0 \mathrm{MPa}$. As shown in Fig. 2a, $5.0 \%$ of the die insert becomes plastic. The figure also presents residual stresses and residual displacement distribution on the combined die obtained from simulation results.

Meanwhile, the residual stresses and residual displacement in the autofrettaged double-layer combined die were calculated on a theoretical basis using the above formulas. As shown in Fig. 3, the radial residual stress is compressive stress, and it reduces with the radius of the die insert but increases with the radius of the stress ring. The circumferential residual stress on the die insert increases first rapidly then slowly with the radius, and the circumferential residual stress on the stress ring becomes tensile stress and decreases with the radius. The distributions of the residual stresses agree perfectly with the results obtained from FE simulation.

The residual stresses and displacements at the die boundaries are further extracted and listed in Table 2. The difference between theoretical and simulation values of the radial residual stress is $10.3 \mathrm{MPa}$ at the inner diameter of the die insert, and the difference is only $0.8 \mathrm{MPa}$ at the outer diameter of the stress ring. The absolute error of the values at the interface is less than $16.11 \%$. In the case of the circumferential residual stress, the absolute error between simulation and theoretical values is $5.62-9.49 \%$. The values of residual 

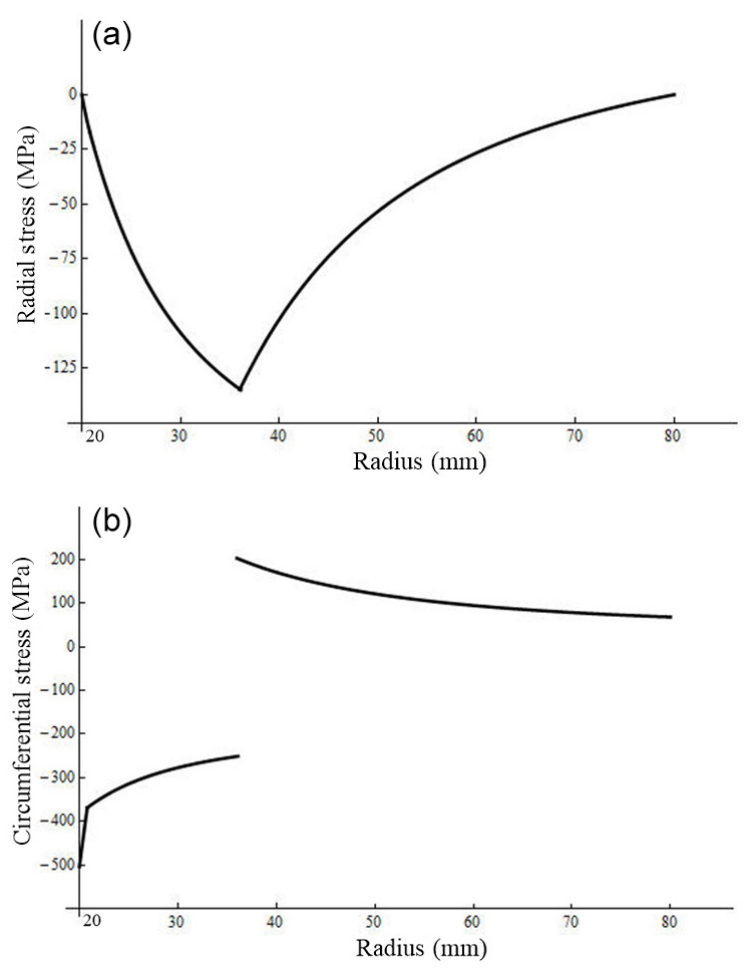

Figure 3. Distributions of (a) radial and (b) circumferential residual stresses obtained from theoretical calculation.

displacement at the inner and outer diameters of the insert die and stress ring are the same. There is good agreement between the theoretical calculations and FE simulation results.

\section{Numerical results and discussion}

For further analysis, nine cases of the combined die strengthened by the autofrettage process were numerically solved using the theory and formulas given in Sect. 2. The cases are listed in Table 3. The table gives important parameters, including the ratio of the plastic area and the yield strength of the die insert. The outer diameters of the die insert and stress ring were chosen as variables. Different ratios of the plastic area meant that there were different yield radii, requiring different autofrettage pressures. Here, the required autofrettage pressure was between 823.1 and $1144.4 \mathrm{MPa}$, which is a range of pressures that can be applied in practice (http://www.felss.com). The inner diameter of the stress ring should increase as the outer diameter of the die insert decreases.

For comparison, the case of the conventional combined die was chosen as case $\mathrm{X}$, and the relative stresses were solved using the typical Lamé equation.

\subsection{Effect of the ratio of the plastic area}

The ratio of the plastic area on the die insert is the main index that reflects the effect of the autofrettage process. In cases I, II and III, the ratio of the plastic area varies from 3 to $8 \%$, and the results of residual stresses in these cases are given in Table 4. The residual stresses on the die insert are compressive, which improves the strength of the die insert. However, the circumferential residual stresses on the stress ring are tensile, which is not beneficial for the stress ring.

The absolute values of radial residual stresses slightly increase with the ratio of the plastic area. However, the value of the circumferential residual stress at the inner diameter of the die insert increases with the ratio of the plastic area. The compressive residual stress at the internal boundary of the die insert after autofrettage is much larger than that in the conventional combined die, which can better offset the tensile stress caused by the subsequent working process. Therefore, the autofrettage process can reduce the risk of longitudinal cracking on the internal surface of the die insert.

When the ratio of the plastic area reaches $8 \%$, the circumferential residual stress near the internal surface of the die insert is $-572.0 \mathrm{MPa}$, which is around 1.5 times that generated in the conventional combined die.

At the same time, the circumferential residual stress near the internal surface of the stress ring increases to $204.3 \mathrm{MPa}$, and the increase in amplitude is $1.6 \%$ relative to the conventional combined die. The greater circumferential tensile stress can increase the failure of the stress ring.

\subsection{Effect of yield strength}

In the forging process, the strength of the die steel is an important factor affecting the die life. In the case studies, the yield strength of the stress ring is $900 \mathrm{MPa}$ and three different tool steels with yield strengths of 1100,1300 and $1650 \mathrm{MPa}$ are selected as the material of the die insert. Therefore, theoretical calculations were made for the different yield strengths of the die insert, and the results are presented in Table 5.

Table 5 shows that the radial residual stress changes little with a variation in yield strength. Meanwhile, the circumferential residual stress on the die insert increases with the yield strength. As the yield strength increases from 1100 to $1650 \mathrm{MPa}$, the circumferential compressive stress near the internal surface of the die insert increases by about $10.34 \%$ and reaches $535.6 \mathrm{MPa}$, while the circumferential tensile stress near the internal surface of the stress ring remains almost the same.

In the case of the conventional combined die, prestress does not affect the yield strength of the die insert and stress ring because the prestressing behavior is a kind of elastic deformation effect originating from the interference fit. The residual stress is much smaller than that of the combined die after the autofrettage process. The circumferential residual 
Table 2. Residual stresses and displacements at die boundaries.

\begin{tabular}{llrr|rr}
\hline & Calculation methods & Die insert & \multicolumn{2}{c}{ Stress ring } \\
\cline { 3 - 6 } & & $r_{1}$ & $r_{2}$ & $r_{3}$ & $r_{4}$ \\
\hline Radial residual stress (MPa) & theoretical & 0 & -134.7 & -134.7 & 0 \\
& simulation & 10.3 & -121.5 & -113.0 & -0.8 \\
\hline Circumferential residual stress (MPa) & theoretical & -503.6 & -250.7 & 202.8 & 68.1 \\
& simulation & -475.3 & -226.9 & 187.6 & 61.9 \\
\hline Residual displacement (mm) & theoretical & -0.03 & -0.03 & 0.04 & 0.02 \\
& simulation & -0.03 & -0.03 & 0.04 & 0.02 \\
\hline
\end{tabular}

Table 3. Parameters of cases selected for further analysis.

\begin{tabular}{|c|c|c|c|c|c|c|c|c|c|}
\hline \multirow[t]{2}{*}{ Case } & \multicolumn{4}{|c|}{ Die insert } & \multirow{2}{*}{$\begin{array}{r}\text { Stress ring } \\
\text { Yield strength } \\
(\mathrm{MPa})\end{array}$} & \multicolumn{2}{|c|}{ Die insert } & \multicolumn{2}{|c|}{ Stress ring } \\
\hline & $\begin{array}{r}\text { Ratio of } \\
\text { plastic area }\end{array}$ & $\begin{array}{r}\text { Yield radius } \\
(\mathrm{mm})\end{array}$ & $\begin{array}{r}\text { Autofrettage } \\
\text { pressure }(\mathrm{MPa})\end{array}$ & $\begin{array}{r}\text { Yield strength } \\
(\mathrm{MPa})\end{array}$ & & $\begin{array}{r}r_{1} \\
(\mathrm{~mm})\end{array}$ & $\begin{array}{r}r_{2} \\
(\mathrm{~mm})\end{array}$ & $\begin{array}{r}r_{3} \\
(\mathrm{~mm})\end{array}$ & $\begin{array}{r}r_{4} \\
(\mathrm{~mm})\end{array}$ \\
\hline I & $3 \%$ & 20.48 & 918.1 & 1300 & 900 & 20 & 36.07 & 36 & 80 \\
\hline II & $5 \%$ & 20.80 & 940.0 & 1300 & 900 & 20 & 36.07 & 36 & 80 \\
\hline III & $8 \%$ & 21.29 & 972.0 & 1300 & 900 & 20 & 36.07 & 36 & 80 \\
\hline IV & $5 \%$ & 20.80 & 823.1 & 1100 & 900 & 20 & 36.07 & 36 & 80 \\
\hline V & $5 \%$ & 20.80 & 1144.4 & 1650 & 900 & 20 & 36.07 & 36 & 80 \\
\hline VI & $5 \%$ & 20.75 & 944.4 & 1300 & 900 & 20 & 35.07 & 36 & 80 \\
\hline VII & $5 \%$ & 20.70 & 949.2 & 1300 & 900 & 20 & 34.07 & 36 & 80 \\
\hline VIII & $5 \%$ & 20.80 & 926.7 & 1300 & 900 & 20 & 36.07 & 36 & 75 \\
\hline VX & $5 \%$ & 20.80 & 910.5 & 1300 & 900 & 20 & 36.07 & 36 & 70 \\
\hline$X$ & - & - & - & 1300 & 900 & 20 & 36.07 & 36 & 80 \\
\hline
\end{tabular}

stress at the inner surface is only $79.30 \%$ of that generated from the autofrettage process even when the yield strength of the die insert is the minimum $1100 \mathrm{MPa}$.

Taking the increase in circumferential residual stress as an index, it is seen that an increase in the ratio of the plastic area of the die insert is more effective than a change to a die material with higher yield strength. As shown in case V, the required autofrettage pressure reaches 1144.4 MPa when the yield strength of the die insert is $1650 \mathrm{MPa}$, and such pressure is difficult to realize in an actual autofrettage process. Therefore, a die insert made of lower-strength material could be used in the autofrettaged die to obtain the good effect of the prestress state.

\subsection{Effect of die dimensions}

In further study, the effect of die dimensions on the combined die after autofrettage was investigated. Both the residual stresses after autofrettage and the stresses during the working process were calculated, and the values at the inner diameters of the die insert and stress ring, which correspond to the zone most likely to fail during the working process, are listed in Table 6. The working pressure is assumed as $900 \mathrm{MPa}$, and the equivalent stress during the working process is computed to evaluate the die failure. Gener- ally, the die will fail when the equivalent stress reaches the yield strength of the die material. In the cases considered, the working stresses are equal to the sum of residual stresses from autofrettage and the new stresses generated by the assumed working pressure. The equivalent stress is simply calculated according to the von Mises yield criterion as

$\bar{\sigma}=\frac{\sqrt{3}}{2}\left(\sigma_{\theta}-\sigma_{r}\right)$

In the case of the conventional combined die, the maximum working pressure is determined as $884.3 \mathrm{MPa}$ when the equivalent stress of the die insert reaches a yield strength of $1300 \mathrm{MPa}$ according to Lamé's formula and the von Mises criterion. Compared with the conventional combined die, the autofrettaged combined die can bear larger working pressure, as expected.

In cases VI and VII, the outer diameter of the die insert is 35.0 and $34.0 \mathrm{~mm}$, respectively, and the variation in residual stress after autofrettage is within 10.2 MPa. During the working process, the circumferential stresses of the die insert become tensile stresses, and the stress of the stress ring increases with decreasing inner diameter of the ring. The equivalent stress on the internal surface of the die insert hardly changes and is far below the yield strength. However, when the outer diameter of the die insert is reduced to 
Table 4. Residual stresses in cases with different ratios of the plastic area.

\begin{tabular}{llrrr|rr}
\hline \multirow{2}{*}{ Stress } & Case & \multicolumn{3}{c|}{ Die insert } & \multicolumn{2}{c}{ Stress ring } \\
\cline { 3 - 7 } & & $r_{1}$ & $r_{p}$ & $r_{2}$ & $r_{3}$ & $r_{4}$ \\
\hline Radial residual stress (MPa) & I & 0 & -9.9 & -134.3 & -134.3 & 0 \\
& II & 0 & -17.0 & -134.7 & -134.7 & 0 \\
& III & 0 & -28.2 & -135.7 & -135.7 & 0 \\
& X & 0 & - & -133.3 & -133.3 & 0 \\
\hline Circumferential residual stress (MPa) & I & -457.1 & -375.7 & -251.3 & 202.2 & 67.9 \\
& II & -503.6 & -368.4 & -250.7 & 202.8 & 68.1 \\
& III & -572.0 & -356.8 & -249.3 & 204.3 & 68.6 \\
& X & -384.9 & - & -251.6 & 201.0 & 67.7 \\
\hline
\end{tabular}

Table 5. Residual stresses in cases with different yield strengths.

\begin{tabular}{|c|c|c|c|c|c|c|}
\hline \multirow[t]{2}{*}{ Stress } & \multirow[t]{2}{*}{ Case } & \multicolumn{3}{|c|}{ Die insert } & \multicolumn{2}{|c|}{ Stress ring } \\
\hline & & $r_{1}$ & $r_{p}$ & $r_{2}$ & $r_{3}$ & $r_{4}$ \\
\hline \multirow[t]{4}{*}{ Radial residual stress (MPa) } & IV & 0 & -16.6 & -134.5 & -134.5 & 0 \\
\hline & II & 0 & -17.0 & -134.7 & -134.7 & 0 \\
\hline & $\mathrm{V}$ & 0 & -17.6 & -135.0 & -135.0 & 0 \\
\hline & $\mathrm{X}$ & 0 & - & -133.3 & -133.3 & 0 \\
\hline \multirow[t]{4}{*}{ Circumferential residual stress (MPa) } & IV & -485.4 & -368.7 & -250.8 & 202.6 & 68.1 \\
\hline & II & -503.6 & -368.4 & -250.7 & 202.8 & 68.1 \\
\hline & $\mathrm{V}$ & -535.6 & -367.9 & -250.5 & 203.3 & 68.3 \\
\hline & $\mathrm{X}$ & -384.9 & - & -251.6 & 201.0 & 67.7 \\
\hline
\end{tabular}

$34.0 \mathrm{~mm}$, the equivalent stress of the stress ring increases to 864.7 MPa, which is close to the yield strength of $900 \mathrm{MPa}$. From a different view, a smaller die insert can be adopted after autofrettage to reduce the amount of expensive material that is usually used for the die insert of the conventional combined die. Taking case VII as an example, more than $15.0 \%$ of the material used for the die insert can be saved.

In cases VIII and VX, the outer diameter of the stress ring is reduced to 75.0 and $70.0 \mathrm{~mm}$, while the residual stresses after autofrettage increase. During the working process, the increase in amplitude of the equivalent stress near the inner surface of the die insert is larger than that of the stress ring. When the outer diameter of the stress ring is reduced to $70.0 \mathrm{~mm}$, the equivalent stress of the die insert increases to 1280.3 MPa, and is very close to the yield strength of the die insert. Therefore, the autofrettaged die with a smaller outerdiameter stress ring can be used to substitute the conventional combined die, resulting in a material savings. More importantly, working space can be saved if the outer diameter of the stress ring can be reduced, especially for multi-stage forging dies.

\section{Conclusions}

1. To improve the strength of the combined die, an alternative method of designing the combined die was proposed to introduce compressive stresses on the die insert through the autofrettage process. The analytical solution for the autofrettage process of a double-layer combined die was obtained. The relationship between the autofrettage pressure and the yield radius of the die insert was determined, and expressions of residual stresses and displacements directly related to geometric parameters, material properties and internal pressure were derived. This theoretical derivation can guide the design of the autofrettaged die.

2. A specific case of a double-layer combined die strengthened in the autofrettage process was simulated employing the FE method. In the case of radial residual stress, the absolute error between simulation and theoretical values at the interface between the die insert and stress ring is less than $16.11 \%$. In the case of the circumferential residual stress, the absolute error is 5.62-9.49\%. In the case of the residual displacement, simulation and theoretical values are the same. The good agreement between theoretical calculations and FE simulation results 
Table 6. Results for different external diameters of the insert die and stress ring.

\begin{tabular}{|c|c|c|c|c|c|c|c|c|}
\hline Process & Stress & Position & VII & VI & II & VIII & VX & $\mathrm{X}$ \\
\hline \multirow[t]{4}{*}{ After autofrettage process } & Radial residual stress (MPa) & $r_{1}$ & 0 & 0 & 0 & 0 & 0 & 0 \\
\hline & & $r_{3}$ & -138.7 & -136.9 & -134.7 & -131.2 & -126.8 & -133.3 \\
\hline & Circumferential residual stress (MPa) & $r_{1}$ & -523.2 & -513.0 & -503.6 & -493.6 & -481.0 & -384.9 \\
\hline & & $r_{3}$ & 199.5 & 201.4 & 202.8 & 209.4 & 217.7 & 201.0 \\
\hline \multirow[t]{7}{*}{ Working process } & Working pressure (MPa) & \multicolumn{6}{|c|}{900} & 884.3 \\
\hline & \multirow[t]{2}{*}{ Radial stress (MPa) } & $r_{1}$ & -900.0 & -900.0 & -900.0 & -900.0 & -900.0 & -884.3 \\
\hline & & $r_{3}$ & -409.1 & -388.7 & -369.5 & -359.9 & -347.9 & -364.0 \\
\hline & \multirow[t]{2}{*}{ Circumferential stress (MPa) } & $r_{1}$ & 496.3 & 506.5 & 515.9 & 543.7 & 578.4 & 616.8 \\
\hline & & $r_{3}$ & 589.4 & 572.7 & 557.2 & 575.4 & 598.1 & 549.1 \\
\hline & \multirow[t]{2}{*}{ Equivalent stress (MPa) } & $r_{1}$ & 1209.2 & 1218.0 & 1226.2 & 1250.3 & 1280.3 & 1300.0 \\
\hline & & $r_{3}$ & 864.7 & 832.7 & 802.5 & 809.9 & 819.2 & 790.8 \\
\hline
\end{tabular}

verifies the reliability and accuracy of the theoretical derivation.

3. In further analysis, nine cases of the combined die strengthened in the autofrettage process were numerically solved using the theoretical derivation and four key parameters, namely the ratio of the plastic area and the yield strength of the die insert and the outer diameters of the die insert and stress ring, and the effects of the parameters on the autofrettage effect were investigated. For comparison, the relative stresses in the case of the conventional combined die were solved using the typical Lamé equation.

4. The circumferential residual stress at the inner diameter of the die insert increases with the ratio of the plastic area and the yield strength of the die insert. Taking the increase in the circumferential residual stress at the inner diameter of the die as an index, it was seen that an increase in the ratio of the plastic area of the die insert is more effective than a change to a die material with higher yield strength.

5. Compared with the conventional combined die, the autofrettaged die could bear larger working pressure, as expected. The autofrettaged die with a smaller-outerdiameter die insert could be used to substitute the conventional combined die, reducing the amount of expensive material required for the die insert. The autofrettaged die with a smaller-outer-diameter stress ring could be an alternative design to the conventional die, resulting in more working space, especially for multi-stage forging dies.

Data availability. The FE model data are available in the Supplement.

The Supplement related to this article is available online at https://doi.org/10.5194/ms-8-267-2017-supplement.
Competing interests. The authors declare that they have no conflict of interest.

Acknowledgements. This work was supported by the National Natural Science Foundation of China (no. 51475294).

Edited by: Chin-Hsing Kuo

Reviewed by: Uday Shanker Dixit and two anonymous referees

\section{References}

Anantharam, K. and Kumar, B. S. K.: Design analysis of high pressure cylinders subjected to autofrettage process using MATLAB and ANSYS, Int. J. Eng. Res. Technol., ESRSA Publications, November, 955-961, 2014.

Bhatnagar, R. M.: Modelling, validation and design of autofrettage and compound cylinder, Eur. J. Mech. A-Solid, 39, 17-25, 2013.

Groenbaek, J. and Birker, T.: Innovations in cold forging die design, J. Mater. Process Tech., 98, 155-161, 2000.

Hojjati, M. H. and Hassani, A.: Theoretical and finite-element modeling of autofrettage process in strain-hardening thick-walled cylinders, Int. J. Pres. Ves. Pip., 84, 310-319, 2007.

Hur, K. D., Choi, Y., and Yeo, H. T.: Design for stiffness reinforcement in backward extrusion die, J. Mater. Process Tech., 130, 411-415, 2002.

Hur, K. D., Choi, Y., and Yeo, H. T.: A design method for cold backward extrusion using FE analysis, Finite Elem. Anal. Des., 40, 173-185, 2003.

Jahed, H., Farshi, B., and Hosseini, M.: Fatigue life prediction of autofrettage tubes using actual material behaviour, Int. J. Pres. Ves. Pip., 83, 749-755, 2006.

Joun, M. S., Lee, M. C., and Park, J. M.: Finite element analysis of prestressed die set in cold forging, Int. J. Mach. Tool. Manu., 42, 1213-1222, 2002.

Kwan, C. T. and Wang, C. C.: An optimal pre-stress die design of cold backward extrusion by RSM Method, Structural Longevity, 5, 25-32, 2011.

Majzoobi, G. H., Farrahi, G. H., and Mahmoudi, A. H.: A finite element simulation and an experimental study of autofrettage for strain hardened thick-walled cylinders, Mat. Sci. Eng. A-Struct., 359, 326-331, 2003. 
Pedersen, T. Ø.: Numerical modelling of cyclic plasticity and fatigue damage in cold-forging tools, Int. J. Mech. Sci., 42, 799818, 2000.

Qian, L. Y., Liu, Q. K., Han, Y., Li, H., and Wang, W. L.: Research on autofrettage technology for extrusion die based on bilinear hardening model[J], Chin. J. Mech. Eng., 47, 26-31, 2011 (in Chinese).

Son, D. S., Hong, J. H., and Chang, S. H.: Determination of the autofrettage pressure and estimation of material failures of a Type III hydrogen pressure vessel by using finite element analysis, Int. J. Hydrogen Energ., 37, 12771-12781, 2012.

$\mathrm{Xu}$, B. Y. and Liu, X. S.: Applied Elasto-plastic Mechanics, Tsinghua University Press, Beijing, 195 pp., 1995 (in Chinese).
Yang, Q. H., Chen, X., Meng, B., and Pan, J.: Optimum design of combined cold extrusion die for bevel gear[J], IACSIT Int. J. Eng. Technol., 4, 348-351, 2012.

Yeo, H. T., Choi, Y., and Hur, K. D.: Analysis and design of the prestressed cold extrusion die, Int. J. Adv. Manuf. Tech., 18, 5461, 2001.

Zhu, R. L.: Results resulting from autofrettage of cylinder, Chin. J. Mech. Eng.-En., 21, 105-110, 2008a.

Zhu, R. L.: Ultimate load-bearing capacity of cylinder derived from autofrettage under ideal condition, Chin. J. Mech. Eng.-En., 21, 80-87, 2008b. 